\title{
Características y prácticas del soporte nutricional enteral en servicios públicos de salud del Departamento Central - Paraguay
}

\author{
Claudia Bordón Riveros, Ana Aguilar-Rabito, Susana Sánchez Bernal, Laura Mendoza de Arbo
}

Instituto Nacional de Alimentación y Nutrición, Ministerio de Salud Pública y Bienestar Social. Asunción, Paraguay

Cómo referenciar este artículo/

How to reference this article:

\begin{abstract}
Bordón C, Aguilar-Rabito A, Sánchez S, Mendoza L.Características y prácticas del soporte nutricional enteral en servicios públicos de salud del Departamento Central - Paraguay. Mem. Inst. Investig. Cienc. Salud. 2016;14(3):14-23
\end{abstract}

\section{R E S U M E N}

Es conocida la alta prevalencia de desnutrición en pacientes hospitalizados, en los que el soporte nutricional es una herramienta clave de intervención. El objetivo fue describir las características y prácticas del soporte nutricional enteral en servicios de salud públicos del Departamento Central - Paraguay. Estudio observacional descriptivo transversal realizado en servicios de salud públicos del Departamento Central - Paraguay que brindan soporte nutricional enteral. Se aplicó una planilla de verificación para describir la planta física, infraestructura, equipamiento, organización y gestión, garantía de inocuidad y calidad y procedimientos relacionados a la nutrición enteral de los pacientes. Se utilizó como referencia la Guía de buenas prácticas, organización y funcionamiento de establecimientos de producción de fórmulas enterales y servicios de nutrición enteral. Un puntaje $\geq 70 \%$ fue considerado como un cumplimiento esperado. Se evaluaron 26 servicios de salud que fueron los que cumplían con los criterios de inclusión, de los cuales 20 correspondían al Ministerio de Salud Pública y Bienestar Social. Del total, 8 contaban con un espacio físico exclusivo para la preparación de fórmulas enterales; 10 cumplieron con los requisitos mínimos de planta física (de ellos, solo 3 tenían separación física de las áreas); 9 con infraestructura; 7con equipamientos; 4 con la garantía de inocuidad y calidad; 8 con organización y gestión y 11 con los procedimientos relacionados a la nutrición enteral. Solo 6 cumplieron con un puntaje $\geq 70 \%$ (categoría "bueno"). Se observaron debilidades en el soporte nutricional enteral en servicios de salud públicos por lo que urge establecer medidas correctivas.

Palabras claves: soporte nutricional enteral, fórmulas enterales.

\section{Characteristics and practices of enteral nutritional support in public health services of the Central Department - Paraguay}

\begin{abstract}
A B S T R A C T
It is known the high prevalence of malnutrition in hospitalized patients, where nutritional support is a key intervention tool. The objective was to describe the characteristics and practices of the enteral nutritional support in public health services of the Central Department-Paraguay. This was a cross-sectional study carried out in public health services of the Central Department in Paraguay that provide enteral nutritional support. A check list was used to describe the physical structure, infrastructure, equipment, organization and management, safety and quality assurance and procedures related to patient enteral nutrition. For the evaluation, a guide to good manufacturing practices, organization and operation of enteral formulas production establishments and enteral nutrition services was used as a reference. A score $\geq 70 \%$ was considered an expected compliance. Twenty six
\end{abstract}


health services, which met inclusion criteria, were evaluated Twenty of them corresponded to the Ministry of Health. Eight of the total number had exclusive physical space for the preparation of the enteral formulas; 10 met the minimum requirements for physical structure (only 3 had physical separation of areas); 9 had infrastructure; 7equipment; 4 safety and quality assurance; 8 organization and management and 11 procedures regarding the enteral nutrition. Only 6 met $\geq 70 \%$ score ("good" category). Weaknesses were observed in the enteral nutritional support of public health services, therefore to establish corrective actions is urgent.

Keywords: enteral nutrition support, enteral formulas.

\section{INTRODUCCIÓN}

La nutrición es una herramienta fundamental que colabora con los tratamientos de recuperación de los enfermos. Es bien conocido el deterioro del estado nutricional durante la estancia hospitalaria. Estudios en Paraguay muestran una prevalencia de desnutrición de alrededor del 50\%, cifra similar a las encontradas a nivel regional, donde la deficiencia de la gestión y el desconocimiento son identificados como posibles factores causales (1-6).

El "Soporte Nutricional" es un componente del tratamiento médico que incluye la nutrición oral, enteral y/o parenteral. La Nutrición Enteral (NE) se define como "la introducción controlada de nutrientes en el tubo digestivo, especialmente elaborada y formulada para uso por sondas enterales o por vía oral, industrializada o no, utilizada exclusiva o parcialmente para sustituir o complementar una alimentación en pacientes desnutridos o no" (7-9).

La intervención en los pacientes con algún grado de deterioro nutricional es asociada con mejoría en el estado nutricional, ingesta de nutrientes, función física y calidad de vida, reduciendo así la readmisión hospitalaria (10).

Por otro lado, es bien conocido que la NE es usada ampliamente en los pacientes hospitalizados y que puede estar sometida a una diversidad de focos de contaminación. Investigaciones realizadas han encontrado que hasta un $67 \%$ de las fórmulas enterales se encuentran contaminadas luego de su preparación. Los contaminantes provienen mayoritariamente de la planta física, equipamiento y/o manipulación deficiente por falta de capacitación (11).

Actualmente, a nivel nacional existen inconvenientes en el campo de la alimentación y nutrición en hospitales tanto a nivel estructural como organizativo. Muy escasos hospitales cuentan con un área específica destinada a la nutrición (6).

Normativas y guías técnicas nacionales (12-14) incluyen los lineamientos técnicos referidos a la planta física, equipamientos, recursos humanos, normas de higiene, medidas de control y de administración de la NE que debe disponer todo establecimiento y servicio de salud que prepare fórmulas para la nutrición enteral, sean estos públicos o privados.

El cumplimiento de dichos lineamientos es sin duda de suma importancia, puesto que acompañado de una gestión óptima de los recursos, ofrecería mejorías y avances relevantes tanto para el paciente como para todo el Sistema de Salud.

El presente estudio fue realizado con el objetivo de describir las características y prácticas del soporte nutricional enteral en servicios públicos de salud del departamento Central de Paraguay en relación a las normativas y guías vigentes.

Se espera que los datos recabados sirvan para la adecuación de las normativas y elaboración de pautas técnicas para mejorar la calidad del proceso de Soporte Nutricional Enteral a nivel nacional.

\section{MATERIALES Y MÉTODOS}

Durante los meses abril a junio del 2014 se realizó un estudio observacional descriptivo transversal. Fueron incluidos todos los hospitales de mediana y alta complejidad que contaban con un servicio destinado a realizar soporte nutricional enteral a pacientes pertenecientes al Departamento Central-Paraguay.

Fue estructurado y validado para luego ser aplicado por las propias autoras, un formulario ad hoc que contemplaba dos secciones. La primera sección incluía datos relacionados al servicio de salud (complejidad, público/privado, $\mathrm{n}^{\circ}$ de camas, $\mathrm{n}^{\circ}$ pacientes con soporte nutricional enteral, volumen de producción, entre otros datos); y la segunda una planilla de verificación incluida en la guía elaborada por el Instituto Nacional de Alimentación y Nutrición(14), además de otras normativas nacionales e internacionales (15-20). 
Los componentes verificados fueron: planta física, infraestructura, equipamientos, organización y gestión, procedimientos relacionados a la nutrición enteral y aseguramiento de la calidad y garantía de inocuidad. Cada componente contó con un listado de elementos que fueron verificados y se les otorgó un puntaje según fueran a. Indispensables, por su alto riesgo ante el incumplimiento b. Necesarios, considerados de riesgo intermedio y c. Importante, los de bajo riesgo.

Se determinó la calificación de los componentes verificados de las prácticas de soporte nutricional siguiendo el criterio definido en la Tabla 1.

Tabla 1.Calificación según el porcentaje de cumplimiento de requisitos obtenidos.

\begin{tabular}{ll}
\hline Porcentaje de cumplimiento de requisitos obtenido & Calificación \\
\hline 90 a $100 \%$ & Muy bueno \\
70 a $89 \%$ & Bueno \\
$\mathbf{5 0}$ a $69 \%$ & Regular \\
< a $50 \%$ & Sub-estándar
\end{tabular}

Fuente: Adaptado de Ministerio de Salud de la Nación Resolución 1674/2007 "Directrices de Organización y Funcionamiento del Área de Alimentación y Dietoterapia en Establecimientos Asistenciales". Buenos Aires: Ministerio de Salud de la Nación; 2007

Las variables se evaluaron mediante verificación visual. En el caso que no pudieron ser verificados visualmente, se evaluó según referencia del encuestado.

Por otro lado, cuando el requisito no pudo ser evaluado porque no se aplicaba a esa área o no pudo verificarse, se anuló el ítem y se descontó el puntaje correspondiente. El nuevo puntaje total asignado pasó a ser el $100 \%$ para la obtención del porcentaje de cumplimiento.

Se establecieron también variables denominadas "informativas" las cuales describían algunas variables de interés, sin que puntúen.

Los datos fueron digitalizados, procesados y analizados en una planilla electrónica Microsoft Office Excelß 2010.

Se aplicó una prueba de normalidad para evaluar la distribución de los datos cuantitativos. Dependiendo de la distribución de éstos, los datos cuantitativos se expresaron en promedio $(x)$ y desviación estándar $(D E)$ y en mediana y valores máximos y mínimos. En el caso de variables cualitativas se expresaron en frecuencia $(n)$.

\section{RESULTADOS}

Se evaluaron 26 Servicios de Salud de mediana y alta complejidad del Departamento Central pertenecientes al: Ministerio de Salud Pública y Bienestar Social $(n=20)$, Hospital Universitario $(n=1)$, Sanidad Militar y Policial $(n=2)$, Organismos no Gubernamentales $(n=1)$ y el Instituto de Previsión Social $(n=2)$ que realizan soporte nutricional enteral de manera regular.

La mediana encontrada de pacientes asistidos con soporte nutricional oral y/o enteral al día fue de 13 pacientes (mínimo 1-máximo 140). La mayor cantidad de servicios de salud $(n=20)$ asisten entre 1 a 50 pacientes.

La mediana de producción de fórmulas enterales fue de 17,1 L/día (mínimo 1-máximo 150). Mayoritariamente $(n=21)$ los servicios de salud producen entre 10 a $50 \mathrm{~L} /$ día.

En cuanto a la consulta al encargado del servicio en relación a su percepción sobre la asistencia nutricional y la producción de fórmulas enterales, de cada 10 hospitales, 7 refirieron que eran insuficientes, puesto que la demanda supera a la oferta y los insumos son escasos, lo que limita la cobertura de las necesidades. Esta situación fue referida en su mayoría en los servicios de salud que no cuentan con un área específica de elaboración de fórmulas enterales.

\section{Planta física}

De los 26 servicios de salud, 16 contaban con un área física destinada a la preparación de fórmulas, mientras que en los demás se realizaba en otros sectores como: el área de enfermería, la sala de internados, la cocina y/o el consultorio de nutrición. 
De los que contaban con un área física destinada para la preparación de fórmulas, solo 8 correspondían a una dependencia exclusiva con infraestructura física básica (paredes o mamparas) para preparación de fórmulas.

En cuanto a la ubicación, fue adecuada en $n=12$ (accesible a servicios clínicos, lejos de lugares muy contaminados).

Así también, solo 7 seguían un flujo unidireccional de actividades y 3 presentaban una separación física de las áreas de trabajo (mínimamente área limpia/área sucia). Estos datos pueden visualizarse en la tabla 2.

Tabla 2. Adecuación de los Servicios de salud a los criterios técnicos verificados en el componente planta física $(n=26)$

\begin{tabular}{lll}
\hline Criterios técnicos del componente planta física & Si (n) & No(n) \\
\hline Existencia de un área física destinada a la preparación de fórmulas & 16 & 10 \\
$\begin{array}{l}\text { Dependencia exclusiva con infraestructura física básica para preparación de } \\
\text { fórmulas }\end{array}$ & 8 & 18 \\
Ubicación & 12 & 14 \\
Flujo unidireccional de actividades & 7 & 19 \\
Separación física de las áreas & 3 & 23 \\
\hline
\end{tabular}

Fuente: INAN/MSPBS.

\section{Infraestructura y equipamientos}

Al verificar la infraestructura de los servicios, las no conformidades y las necesidades de equipamientos se detallan en la tabla3.

Tabla 3. Adecuación de los Servicios de salud a los criterios técnicos verificados en el componente infraestructura $(n=26)$

\begin{tabular}{lll}
\hline Criterios técnicos del componente infraestructura & Si (n) & No (n) \\
\hline Pisos en buen estado & 18 & 8 \\
Uniones redondeadas entre los planos & 12 & 14 \\
Aberturas exteriores con protección antiplagas & 18 & 8 \\
Existencia de aire acondicionado & 18 & 8 \\
Iluminación con protección & 10 & 16 \\
Grifos con agua fría y caliente & 13 & 13 \\
Llave accionada con el codo & 0 & 26 \\
Registros de controles de potabilidad del agua & 15 & 11 \\
Basurero con pedal & 8 & 18 \\
Recordatorios de lavado de mano & 7 & 19 \\
Disponibilidad de jabón y toallas desechables & 10 & 16 \\
Autoclave & 3 & 23 \\
Carros o material para la distribución & 18 & 8 \\
Balanza digital & 20 & 6 \\
Ollas/cacerolas de acero inoxidable exclusivos & 14 & 12 \\
Contenedores aptos y exclusivos para alimentación enteral & 14 & 12 \\
Mesada lisa de material adecuado & 19 & 7 \\
Licuadora exclusiva & 20 & 6 \\
Bacha de acero inoxidable exclusivo & 14 & 12 \\
Jarros graduados exclusivos & 14 & 12 \\
Toallas y jabón líquido en el lugar de preparación & 10 & 16 \\
Cartel de recordatorio de lavado de manos & 7 & 19 \\
Muebles para la oficina de nutrición & 17 & 9 \\
Sistema de informatización en la oficina de nutrición & 17 & 9 \\
\hline Fuente NANMSPS. & & \\
\hline
\end{tabular}

Fuente: INAN/MSPBS. 


\section{Organización y gestión}

El área de nutrición figuraba en el organigrama funcional en 17 de los 26 Servicios de Salud (aunque en varios de ellos solo como propuesta, no aprobada aún). Se encontró falta de registros que establecen y definen las responsabilidades de cada individuo $(n=8)$, como así también protocolos de manejo $(n=13)$.

En cuanto al total de nutricionistas, 19 servicios de salud disponían de la cantidad necesaria de profesionales en el área clínica (en el caso en que la función era exclusivamente nutrición clínica). La conformación de equipos de terapia nutricional (mínimamente licenciado en nutrición, médico y licenciado en enfermería capacitado) se observó en una minoría de los casos $(n=5)$.

En general, las capacitaciones se realizaban por autogestión $(n=11)$ y no se contaban con registros de participación en las mismas $(n=20)$.

De los 26 Servicios de Salud, 17 disponían de fórmulas industrializadas para la mayor cantidad de los pacientes que la necesitaban y 14 refirieron que suele ser sostenible en el tiempo la disponibilidad de las fórmulas industrializadas. Sin embargo, la fuente de aprovisionamiento predominante de estos insumos eran los familiares $(n=16)$ y las donaciones $(n=17)$. Solo en 7 Servicios de Salud, el servicio proveía dichos insumos. En algunos casos refirieron utilizar fórmulas artesanales para los pacientes $(n=14)$.

El número de servicios de salud que se adecuan según el componente organización y gestión se describe en la tabla 4.

Tabla 4. Adecuación de los Servicios de salud a los criterios técnicos verificados en el componente organización y gestión $(n=26)$

\begin{tabular}{lll}
\hline Criterios técnicos del componente organización y gestión & Si (n) & No (n) \\
\hline Disposición de organigrama del área de nutrición & 17 & 9 \\
Manual de funciones & 18 & 8 \\
Protocolos de trabajo & 13 & 13 \\
Disponibilidad de nutricionista & 19 & 7 \\
Capacitación & 11 & 15 \\
Registro de capacitación & 6 & 20 \\
Disponibilidad de fórmulas enterales & 17 & 9 \\
\hline Fuente: INAN/MSPBS & &
\end{tabular}

\section{Garantía de inocuidad y calidad}

Los procedimientos operacionales para todas las operaciones críticas que involucran el soporte nutricional (flujogramas de procesos) no estaban estandarizados en 20 de los hospitales.

En 18 servicios no se tenían identificados los Puntos Críticos de Control (PCC) de los procedimientos, clave para el aseguramiento de la inocuidad. Solo algunos refirieron llevar trazabilidad $(n=6)$, programa de higiene $(n=7)$, monitoreo microbiológico en el área de producción $(n=5)$ y de las fórmulas $(n=3)$.

Otros criterios técnicos que frecuentemente no se lograron fueron la existencia de protocolo para selección de fórmulas $(n=8)$, mantenimiento en el mismo contenedor desde la elaboración hasta la infusión $(n=19)$, registro de temperatura de equipos de frío $(n=16)$, personal encargado de calidad $(n=19)$ y certificado médico del personal para manipulación de alimentos $(n=9)$.

La tabla 5 describe el número de servicios de salud adecuados en cada criterio técnico evaluado del componente garantía de inocuidad y calidad. 
Tabla 5. Adecuación de los Servicios de salud a los criterios técnicos verificados en el componente garantía de inocuidad y calidad $(n=26)$

\begin{tabular}{lll}
\hline Criterios técnicos del componente garantía de inocuidad y calidad & Si (n) & No (n) \\
\hline Estandarización de procedimientos operacionales & 6 & 20 \\
Identificación de puntos críticos & 8 & 18 \\
Trazabilidad & 6 & 20 \\
Programa de higiene & 7 & 19 \\
Monitoreo microbiológico en el área de producción & 5 & 21 \\
Monitoreo microbiológico de las fórmulas & 3 & 23 \\
\hline
\end{tabular}

Fuente: INAN/MSPBS.

\section{Procedimientos relacionados a la nutrición enteral}

La mayoría realizaba visitas diarias a los pacientes con soporte nutricional $(n=21)$.

Se refirió que utilizan como herramienta de evaluación la Valoración Global Subjetiva $(n=11)$ y la antropometría $(n=12)$. La indicación de la nutrición enteral era efectuada por un médico conjuntamente con el nutricionista y existían registros de las prescripciones en la ficha clínica en 24 servicios.

Si utilizaban fórmulas en polvo, el nutricionista efectuaba los cálculos necesarios. En 12 Hospitales no se destinaba a una persona responsable de la preparación, por lo que dicha actividad lo realizaba enfermería, familiar o nutricionista.

La preparación y la manipulación de las fórmulas no se elaboraban con técnicas asépticas en 17 de los hospitales y en 9 no se desinfectaban los utensilios antes del uso.

En algunos casos $(n=6)$ se refrigeraban las fórmulas por un tiempo mayor a 12 horas, lo que podría favorecer a la proliferación de microorganismos.

Una vez preparadas las fórmulas enterales no se transportaban en equipos que mantenían la cadena de frío ni se verificaba la integridad de la fórmula en la recepción en 7 hospitales.

En alrededor de la mitad de los hospitales, la enfermera era la que administraba la nutrición enteral, en los demás eran los familiares $(n=15)$ los que realizaban esta actividado en su defecto las nutricionistas $(n=2)$.

Quince servicios de salud refirieron no contar con protocolos de administración de la nutrición enteral, solo se basaban en las indicaciones escritas en la ficha.

\section{Cumplimiento de los componentes técnicos verificados}

En la Tabla 6 se muestra los servicios de salud discriminados según la calificación en cada componente técnico verificado.

En el componente técnico planta física, infraestructura y equipamientos 11 ; 9 y 12 servicios de salud respectivamente obtuvieron una calificación "sub-estándar".

El componente técnico garantía de calidad e inocuidad fue el componente en el cual 16 servicios de salud alcanzaron la categoría de "sub-estándar" siendo el componente con mayor incumplimiento.

Por otro lado, tanto en el componente de organización y gestión como en el de procedimientos relacionados a la nutrición enteral, 9 servicios de salud obtuvieron la calificación de regular.

Teniendo en cuenta la suma de todos los componentes, la mayor cantidad de servicios de salud obtuvo una calificación "regular" $(n=11)$ y "sub-estándar" $(n=9)$, quedando solo 6 servicios con la categoría "bueno" y ninguno "muy bueno". 
Tabla 6. Servicios de salud según el puntaje del componente técnico verificado $(n=26)$

\begin{tabular}{llllll}
\hline \multirow{2}{*}{ Componente técnico verificado } & \multicolumn{5}{c}{ Servicio de salud (n) } \\
\cline { 2 - 6 } & $\begin{array}{l}\text { Muy } \\
\text { bueno }\end{array}$ & Bueno & Regular & $\begin{array}{l}\text { Sub- } \\
\text { estándar }\end{array}$ & TOTAL \\
\hline Planta física & 3 & 7 & 5 & 11 & 26 \\
Infraestructura & 1 & 8 & 8 & 9 & 26 \\
Equipamientos & 1 & 6 & 7 & 12 & 26 \\
Garantía de inocuidad y calidad & 2 & 2 & 6 & 16 & 26 \\
Organización y gestión & 2 & 6 & 9 & 9 & 26 \\
Procedimientos relacionados a la nutrición & 2 & 9 & 9 & 6 & 26 \\
enteral & 0 & 6 & 11 & 9 & 26 \\
Suma total de los componentes & & & & &
\end{tabular}

Fuente: INAN/MSPBS.

\section{DISCUSIÓN}

En la elaboración de los criterios técnicos establecidos, se utilizaron normativas, publicaciones y recomendaciones nacionales e internacionales. Todas ellas, como factor crítico indispensable, establecen que las instituciones que manipulan fórmulas enterales en polvo imprescindiblemente deberán contar con un área física delimitada para preparación exclusiva de las mismas (12-14,21-26).

En la presente investigación se evidenció la falta de espacio físico destinado para ese fin ya que solo 8 de los 26 servicios de salud público de mayor complejidad de Paraguay a nivel central cuentan con el mismo. Así también se recomienda que la planta física cuente con áreas diferenciadas con el fin de proporcionar áreas de contaminación controlada; sin embargo, se encontró que solo 3 cumplieron con dicha recomendación.

Es preocupante también el bajo grado de cumplimiento en infraestructura y equipamientos. Un punto crítico encontrado fue la falta de disponibilidad de materiales de aseo, limpieza y desinfección, tanto en las áreas de manipulación como en los vestuarios y sanitarios. La contaminación de los preparados de nutrición enteral se produce, la mayoría de las veces, al ser manipulados por diversos motivos (reconstitución, dilución, incorporación al contenedor), con lo que se aumenta el riesgo de contaminación al aumentar el número de manipulaciones. Así, las infecciones relacionadas con la atención sanitaria (IRAS) suponen una tremenda carga de enfermedad y tienen un importante impacto económico en los pacientes y los sistemas sanitarios de todo el mundo. Según la Organización Mundial de la Salud (OMS), una buena higiene de las manos puede salvar vidas, por lo que además de contar con estos insumos, es necesaria la capacitación constante del personal sanitario relacionado directa 0 indirectamente con el paciente $(27,28)$.

Llamó la atención la necesidad de contar con contenedores aptos y específicos para la nutrición enteral ya que la utilización de botellas de plásticos reutilizadas podría ser también un factor crítico de contaminación. Estudios han mostrado que la mayor fuente de contaminación de las fórmulas preparadas con materia prima en polvo se produciría en la etapa de elaboración por manipulación o utensilios contaminados $(29,30)$.

Así también dentro del proceso de preparación de las fórmulas, la American Society of Enteral and Parenteral Nutrition (ASPEN) identifica que la manipulación de las fórmulas es un punto crítico de control para la prevención de la contaminación (30). Por tanto, la literatura, avala y recomienda la importancia de los sistemas de registros, protocolos, monitoreo de la calidad de las fórmulas a través de los registros de trazabilidad y los manuales de calidad respectivos (31). Estos registros fueron encontrados en la minoría de los servicios de salud evaluados.

Por otro lado, no se cuenta con controles de potabilidad del agua que se utiliza tanto para la preparación como para el lavado de los equipos. En ninguno se utiliza agua filtrada mediante ósmosis inversa. Esta es una no conformidad ya que en todas las normas se recomienda que el agua a utilizar sea potable, segura, filtrada y estéril, pues el agua potable, aunque es sanitizada, pudiera contener patógenos y alto contenido de minerales que pueden, a su vez, alterar los componentes de las fórmulas $(13,14,30)$. 
Así también, un punto preocupante es el tiempo de colgado de las fórmulas, ya que varios servicios no cuentan con protocolos de infusión y monitoreo y además la temperatura promedio en Paraguay es de $22,9^{\circ} \mathrm{C}$, pudiendo llegar a valores de media máxima absoluta de 31 a $41^{\circ} \mathrm{C}(32)$. Se recomienda que las fórmulas elaboradas con materia prima en polvo no estén colgadas por más de cuatro horas y 24 horas para las listas para usar, a una temperatura ambiente, sobre todo las fórmulas con agregados de módulos(30).

En cuanto a la organización y la gestión, se destaca la necesidad de contar con manuales de funciones, procedimientos y protocolos de manejo, considerados indispensables para asegurar la calidad de las preparaciones. Los resultados reflejan un déficit en la gestión del área de nutrición, empezando por una falta en la organización, desde el punto de que "nutrición" no está inserto dentro de la estructura organizacional de los servicios, acompañado de una falta de apoyo en la capacitación técnica de los recursos humanos. Estos hallazgos son similares a los encontrados por Centurión A en un análisis de situación realizado en el 2013 en servicios de alimentación y nutrición (6).

Si bien en más de la mitad de los hospitales se contaba con la cantidad necesaria de licenciados en nutrición, en muchos de ellos, sus funciones no eran exclusivas para el área clínica, ya que se encontraban realizando funciones en el servicio de alimentación y/o el área de salud pública a la par del área clínica. Además, dichas cantidades es conveniente discriminarlas según las características del servicio y tipos de pacientes atendidos (33).

Todos los datos observados en la presente investigación son similares a los encontrados por Lara González S et al., donde existe una preocupación por la falta de cumplimiento de criterios técnicos como el de planta física, áreas de trabajo, equipamientos, inocuidad, recursos humanos capacitados, entre otros (15).

Como fortalezas del trabajo se puede resaltar la relevancia del mismo, puesto que no se contaba con datos similares a nivel nacional. Con los datos recabados se establecerá una línea de base y se pretende que sirvan para actualizar las normativas vigentes, adecuándolas a las necesidades actuales y congeniando con las normativas internacionales.

Como limitación se podría citar que la investigación se circunscribió solamente a un departamento del país, y exclusivamente al ámbito público, por lo que sería de mucha utilidad ampliar los datos a nivel nacional. Además, en la mayoría de los lugares no se contaba con documentación y registros por lo que algunos criterios fueron evaluados según la referencia del encargado del área, disminuyendo posiblemente la validez de esos datos.

Por todo lo expuesto se puede concluir que es alarmante el bajo cumplimiento de los criterios técnicos verificados, sobre todo en el componente de garantía de inocuidad y calidad, ya que estos hospitales son de alta complejidad y atienden a un volumen importante de pacientes, muchos de ellos muy complejos e inmunocomprometidos, por lo que urge establecer medidas correctivas al respecto.

\section{Agradecimientos}

Se agradece a la T.A. Cecilia Martínez, T.A. Rosa Araujo, Lic. Angélica Mena, Lic. Laura Amarilla y Lic. Leticia Leguizamón por su colaboración y participación en el relevamiento de datos y aportes en el instrumento. Un reconocimiento especial a la Lic. Sandra Lara González y al Dr. Eduardo Atalah por sus aportes en cuanto a materiales bibliográficos y experiencias previas. Así mismo a los directores y funcionarios de los hospitales por el acompañamiento.

\section{REFERENCIAS BIBLIOGRAFICAS}

1. Correia MI, Campos A, ELAN Cooperative Study. Prevalence of Hospital Malnutrition in Latin America: the Multicenter ELAN Study. Nutrition 2003;19(10):823-5.

2. Castillo JC, Figueredo R, Dugloszewski C, Díazo JA, Spolidoro JV, Matos A et al. Declaración de Cancún: declaración internacional de Cancún sobre el derecho a la nutrición en los hospitales. Nutr Hosp. $2008 ; 23(5): 413-7$.

3. de Ulibarri J, Picón MJ, García E, Mancha A. Detección precoz y control de la desnutrición hospitalaria. Nutr Hosp. 2002;17:139-46.
4. Waitzberg D, Ravacci G, Raslan M. Desnutrición hospitalaria. Nutr Hosp. 2001;26(2):254-64.doi: 10.1590/S021216112011000200003.

5. Goiburú ME, Alfonzo LF, Aranda AL, Riveros MF, Ughelli MA, Dallman Det al. Nivel de conocimiento en nutrición clínica en miembros del Equipo de Salud de Hospitales Universitarios del Paraguay. Nutr Hosp. 2006; 21(5):591-5.

6. Centurión A. Situación de Servicios de Alimentación y Nutrición en Hospitales Públicos del Paraguay [Monografía de Especialidad]. Asunción-Paraguay: Universidad Nacional de Asunción; 2014. 
7. American Society for Parenteral and Enteral Nutrition Board of Directors and Standars Committee. Definition of Terms, Style, and Conventions Used in ASPEN guideline and standards; $2012 . \quad$ Disponible: http://nutritioncare.org/Professional_Resour ces/Guidelines_and_Standards/Guidēines/2 012_Definitions_of_Terms,_Style,_and_Conv entions_Used_in_A_S_P_E_N_Board_of_Dir ectors-Approved_Documents/

8. Ukleja A, Freeman KL, Gilbert K, Kochevar M, Kraft MD, Russell MK et al. Task Force on Standards for Nutrition Support: Adult Hospitalized Patients, and the American Society for Parenteral and Enteral Nutrition Board of Directors. Nutr Clin Pract. 2010;25(4):403-14.doi: $10.1177 / 0884533610374200$.

9. Lochsa H, Allisonb SP, Meierc R, Pirlicha M, Kondrupd J, Schneidere ST et al. Introductory to the ESPEN Guidelines on Enteral Nutrition: Terminology, definitions and general topics. Clin Nutr. 2006;25(2):180-6.

10. Álvarez J, Peláez N, Muñoz A. Utilización clínica de la Nutrición Enteral. Nutr Hosp. 2006;21(2):87-99.

11. Azario AM. Medidas para la prevención de infecciones en el lactario. ECI. $2010 ; 2(3): 238-44$.

12. Reglamento para la habilitación y funcionamiento de establecimientos dedicados a la preparación, comercialización y distribución de mezclas de nutrición parenteral y de nutrición enteral. Decreto $\mathrm{N}^{\circ}$ 19156/02 de octubre.

13. Sociedad Paraguaya de Nutrición. Normas de Buenas Prácticas para el Soporte Nutricional. Asunción: Sociedad Paraguay de Nutrición; 2001.

14. Instituto Nacional de alimentación y Nutrición - Ministerio de Salud Pública y Bienestar Social. Guía de buenas prácticas, organización y funcionamiento de Establecimientos de producción de fórmulas enterales y servicios de alimentación enteral elaborado por el Instituto Nacional de Alimentación y Nutrición. INAN. En prensa 2016.

15. Lara S, Domecq C, Atalah E. Evaluación de buenas prácticas de manufactura en la elaboración de fórmulas enterales en hospitales públicos de Santiago, Chile. Nutr Hosp. 2013;28(6):2021-6. doi: 10.3305/nutr hosp.v28in06.6864.

16. Duprat G, Coelho W, Akutsu R. Development of a quality control tool for audits in enteral nutrition. Nutr Hosp. 2014;29(1):10220.doi: 10.3305/nh.2014.29.1.7071.

17. Reglamento Técnico de las Condiciones Higiénico-Sanitarias y de Buenas Prácticas de Fabricación para Establecimientos Elaboradores/Industrializadores de Alimentos. Reglamento Técnico del MERCOSUR/GMC/RES No 80/96.

18. Instituto Nacional de Alimentación y Nutrición (INAN). Criterios Técnicos de la Lista de Verificación para Registro de
Establecimientos de Alimentos. Asunción: INAN; 2013. Disponible en: http://inan.gov.py/newweb/?page_id $=504$

19. Ley del Código Sanitario. Ley $N^{\circ} 8 \overline{3} 6 / 80$.

20. Ley que regula el ejercicio de la profesión de los Licenciados en nutrición. Ley $\mathrm{N}^{\circ}$ $3174 / 2007$.

21. Regulamento Técnico os requisitos mínimos exigidos para a Terapia de Nutrição Enteral. Resolução da Diretoria Colegiada $N^{\circ}$ $63 / 2000$.

22. Ministerio de Salud del Gobierno de Chile. Orientación Técnica para Servicios Dietéticos de Leche (Sedile) y Central de Fórmulas Enterales (Cefe). Santiago-Chile: Minsal; 2009.

23. Directrices de Organización y Funcionamiento del Área de Alimentación y Dietoterapia en Establecimientos Asistenciales. Resolución 1674/2007.

24. Dirección de Calidad de los Servicios de Salud. Programa Nacional de Garantía de la Calidad de la Atención Médica. Resolución 168/2007 "Normas de Organización y Funcionamiento de las Áreas". Ministerio de Salud de la Nación. Argentina, 2007.

25. Dirección de Calidad en Servicios de Salud del Ministerio de Salud de la Nación, Sociedad Argentina de Nutrición, Sociedad Argentina de Pediatría, Asociación Argentina de Dietistas y Nutricionistas/Dietistas, Sociedad Argentina de Terapia Intensiva, Asociación de Nutricionistas del Gobierno de Bs.As. Normas de Buenas Prácticas para la Terapia Nutricional Enteral. Argentina, 2004.

26. Ministerio de Salud y Deportes, Instituto Nacional de Seguros de Salud. Guía para la Gestión de Calidad para los Servicios de Alimentación y Nutrición en Establecimiento de Salud de $1^{0}, 2^{\circ}$ y $3^{\circ}$ Nivel de Atención. La Paz-Bolivia: Ministerio de Salud y Deportes; 2008.

27. Lalueza MP, Rodríguez V, Roblez A, Fontán C. Contaminación de nutriciones enterales en pacientes críticos. Validación del proceso de manipulación. Farm Hosp. 1999;23(2):95-102.

28. Organización Mundial de la Salud. Guía de aplicación de la estrategia multimodal de la OMS para la mejora de la higiene de las manos. OMS, 2009.

29. Klaassen J, García P, Maiz A, Campano M. Mecanismos de Contaminación de las Fórmulas para Nutrición Enteral. Rev. chil. infectol. http://dx.doi.org/10.4067/S071610182002000200001.

30. Bakhead R, Boullata J, Brantley S, Corkins M, Guenter P, Krenitsky J et al. Enteral nutrition practice recommendations. JPEN J Parenter Enteral Nutr.2009;33(2):12267.doi: $10.1177 / 0148607108330314$

31. Sociedad Española de Nutrición Parenteral y Enteral. Indicadores de Calidad para las Unidades de Nutrición Clínica. SENPE, 2008. 
32. Dirección Nacional de Aeronáutica Civil. DPTO. DE CLIMATOLOGIA "Centro Meteorológico Nac.-Sajonia". Tablas Climatológicas aeronáutica AISP periodo 2011 - 2015]. Disponible en: http://www.meteorologia.gov.py/publicacion es.php
33. Federación Latinoamericana de Terapia Nutricional y Nutrición Clínica y Metabolismo. Documento de Consenso. Funciones y Competencias del Nutricionista Clínico. FELANPE, 2012. 